JGG 2022;70:134-143

doi: $10.36150 / 2499-6564-N 365$

\title{
Italian long-term care facilities during COVID-19 era: a review
}

\author{
Rossana Gnassoํㄹ Irene lommazzo ${ }^{1}$, Graziamaria Corbi², \\ Francesco Celi ${ }^{1}$, Anna Maria lannicelli ${ }^{3}$, Nicola Ferrara ${ }^{3}$, Carlo Ruosi ${ }^{1}$ \\ ${ }^{1}$ Department of Public Health, University of Naples "Federico II", Naples, Italy; ${ }^{2}$ Department \\ of Medicine and Health Sciences, University of Molise, Campobasso, Italy; Italian Society of \\ Gerontology and Geriatrics (SIGG); ${ }^{3}$ Department of Translational Medical Sciences, University of \\ Naples "Federico II", Naples, Italy
}

Background. Since the start COVID-19 epidemic, 9154 deaths have been registered in Italian Long-Term Care Facilities (LTCF). Half of them were COVID 19-related.

Objective. In this review we analyzed the management of COVID-19 outbreak in Italian LTCF, in the most affected regions.

Results. In the Italian Institute of Health (ISS) report, 1356 LTCF (41.3\%) answered to questionnaires on COVID-19 infection in LTCF. Only $7.4 \%$ of deaths was related to Coronavirus (confirmed by swab), while $33,8 \%$ of deceased presented flu-like symptoms without confirmation by swab. $77.2 \%$ of structures reported the lack of Personal Protective Equipment. The autonomous management policies of the regions cannot be negligible in this assessment, above all Lombardia Region that diverted patients from hospital to extra-structures, like LTCF.

Conclusions. The elderlies represent high-risk patients for developing COVID-19 with rapid clinical deterioration. Containment measures should be particularly optimized, especially in anticipation of another possible pandemic recurrence. Surely a comparison among different systems could help to achieve more information in the standardization of procedures. Identifying flaws in this system is necessary to perfect and optimize epidemic management as much as possible.

Key words: COVID, older persons, long-term care facilities, legislation

\section{Correspondence \\ Graziamaria Corbi \\ Department of Medicine and Health Sciences, University of Molise, Italian Society of Gerontology and Geriatrics (SIGG), via De Sanctis snc, 86100 Campobasso. Tel.: +39087 4404771. Fax +39087 4404778. \\ E-mail: graziamaria.corbi@unimol.it}

How to cite this article: Gnasso R, lommazzo I, Corbi G, et al. Italian longterm care facilities during COVID-19 era: a review. Journal of Gerontology and Geriatrics 2022;70:134-143. https://doi. org/10.36150/2499-6564-N365

(C) Copyright by Società Italiana

di Gerontologia e Geriatria (SIGG)

\section{(c) (1) () $९$}

This is an open access article distributed in accordance with the CC-BY-NC-ND (Creative Commons Attribution-NonCommercial-NoDerivatives 4.0 International) license. The article can be used by giving appropriate credit and mentioning the license, but only for non-commercial purposes and only in the original version. For further information: https://creativecommons.org/licenses/by-nc-nd/4.0/deed.en

\section{INTRODUCTION}

Worldwide, since $31^{\text {st }}$ December 2019 up to $12^{\text {th }}$ May 2020, 413,7193 cases of COVID-19 were registered, including 28,5760 deaths. SARSCoV-2 pandemic had a strong impact on Italy, which was the first European country and one of the first in the World, after China, to be severely affected. In the last report of the SARS-CoV2 Surveillance Group by the Istituto Superiore di Sanità (ISS), along with their geographic distribution, characteristics of 32,938 patients died by SARS-CoV-2 are described ${ }^{1}$. Regions with the highest number of deaths were Lombardia $(16,349$, 49.6\%), Emilia Romagna (4192, 12.7\%), Piemonte (2846, 8.6\%) and Veneto (1964, 6.0\%). Analyzing medical records of 33,350 in-hospital died patients, the mean age of subjects deceased for SARS-CoV-2 infection was 80 years (median 82, range 0-100). 13692 (41.6\%) were women, with a median age higher than men (Women 85 years, Men 79 years). Besides, 
144 subjects (4.2\%) presented no comorbidities, 505 (14.7\%) one, 738 (21.5\%) 2, and 2051 (59.7\%) 33 comorbidities. The most common conditions observed in SARS-CoV-2 positive deceased patients were cardiopulmonary, renal, oncologic, and metabolic diseases $^{1-3}$. A high mortality rate occurred in Long-Term Care Facilities (LTCF), where non-self-sufficient people, like frail older persons, disabled with chronic, neurological diseases, are hosted and assisted. The frail subjects hospitalized in these structures must be considered at higher risk for life when affected by COVID-19.

In this review we analyzed the management of COVID-19 outbreak in Italian LTCF, especially in the most affected regions, hoping to improve the future management under new European guidelines (on $13^{\text {th }}$ May 2020).

\section{ITALIAN LONG-TERM CARE FACILITIES (LTCF) LEGISLATION PRE-COVID-19}

The LCTF provide a medium level of health care (medical, nursing and rehabilitative assistance), and a high level of hotel accommodation, addressed to non-autonomous elders or individuals who cannot receive domiciliary assistance. The Italian Law 67/88 instituted the LCTF to ensure low-intensity medical care and long-residence time that could turn into a permanent stay, depending on the psycho-physical condition of the patients. On the organizational side, the basic unit includes 20-25 spots for non-autonomous older persons and 10-15 for physically and mentally disabled people (depending on the severity of the patient). The organization in blocks allows to accommodate different kinds of patients in the same residential building without conflicts, and provide the necessary discretion, due to the autonomy of each module. At the same time, this lets to socialize with other guests and external users of the daytime service center. This organization in autonomous modular blocks guarantees the best possible care, efficient personnel employment and use of resources ${ }^{4}$. The guests' residential area includes bedrooms available for up to 4 people. According to the DPR no. 384/78 every room must be equipped with a private bathroom; sanitary facilities (at least 1 every 2 rooms for a maximum of 4 guests), must be divided into bathroom and dressing room with washbasin, to ensure better use of these services for the customers, and to make staff's work easier ${ }^{5}$.

\section{WHAT HAPPENED IN ITALIAN LCTF DURING COVID-19 PANDEMIC?}

The final ISS report turns out that 9154 people died,
680 of which resulted positive to a swab test, and 3092 with flu-like symptoms. $7.4 \%$ of the dead subjects had been affected by SARS-CoV-2, 33.8\% exhibited flu-like symptoms without swab. Deaths' peak occurred over the period from 16 to 31 March $2020{ }^{6}$.

The first Italian citizen infected was recorded on $18^{\text {th }}$ February 2020 in Codogno (Lombardia). The DPCM of $4^{\text {th }}$ May 2020 established limited access to healthcare residencies, with prevention measures. Afterward, new regional council decisions were enacted on LCTF management.

The Lombardia Region, the most affected by the pandemic, set the Region Decree no. XI/ $2906^{7}$, which established accommodations from non-hospital buildings to be made available, due to the need for increased use of hospital beds in the intensive care unit (ICU). The measures adopted for this purpose were:

- available non-hospital accommodations check;

- interruption of hospitalization to such facilities after $9^{\text {th }}$ March;

- early hospital discharge;

- interruption of $50 \%$ of LCTF turn over;

- the setting up of a regional center facing calls to discharge from acute care hospitals and finding appropriate target facilities;

- the detection of autonomous facilities with detached pavilions and other sociomedical structures as LCTF for COVID-positive tested patients;

- structures that offer services of integrated home care assistance and professional consultancy services for COVID-positive tested patients ${ }^{7}$.

On $20^{\text {th }}$ March 2020 to control the infection, the Piemonte region highlighted the need to find residential facilities where move COVID-affected patients who don't need hospitalization ${ }^{8}$. Training courses and prevention programs had been developed for health care professionals, to provide professional updates concerning the risks associated with the disease, and the precautionary measures available. The Piemonte region considered essential to empower staff and to have regularly swab done for early detection of infective among hospital staff, providing them protective devices. Furthermore, as a result of the decreasing care for the persons 65 years and older, accompanied by many suspended operations, care services needed to be deployed, and home assistance to be preferred ${ }^{8}$.

To control the infection in LCTF the Toscana region enacted the DR no. 28 of $7^{\text {th }}$ March $2020^{9}$, by which all the positive guests should be taken over by National Health Care Service with the best care pathway, separating those positives or uncertain and asymptomatic cases from the negatives. The Local Health Authority (ASL) have been encouraged to promote the economic enhancement and recruit more staff, particularly if LCTF 
were converted into COVID-positives accommodations.

Moreover, some precautions are set out:

- checks at the entrance of facilities, with denial of access if the people have traveled to the risk-zones in the last 15 days, reported flu-like symptoms, or their access was unnecessary;

- restricted access, only 1 visitor per guest;

- hand hygiene, which includes the use of alcohol-based hand rub (ABHR) both at entry and exit;

- organizing entertainment and socio-educational activities in small groups,

- keeping a distance of at least 1 meter from others;

- ventilation and disinfection of environments;

- staff provided with any single-use safety devices ${ }^{9}$.

The Southern Italian regions also implemented countermeasures for spread of COVID-19 in LTCF. In particular, the Campania region, the most populous region of Southern Italy, on $13^{\text {th }}$ March 2020 issued the Ordinance number 16 which commands the suspension, throughout the regional territory, of the health and welfare activities in all public and private semi-residential territorial health, and social and health services ${ }^{10}$. The Regional Directorate General for the Protection of Health, by the note with the Protocol number 0260863 of $4^{\text {th }}$ June $2020{ }^{11}$, confirmed the indications implemented by the Ministerial Decree of $17^{\text {th }}$ May $2020{ }^{12}$, that allowed the access to relatives and visitors to semi-residential facilities only for cases authorized by the health manager of the structure. Visitors must be screened in advance for the presence of suspicious symptoms for SARS-CoV-2 infection in the 14 days prior to access and equipped with appropriate PPE during their presence in the structure. The regional provision also indicated to limit the entry of new guests and, if there are any, they would have to stop in a separate temporary reception area for 15 days, in order to guarantee an additional barrier against the spread of SARS-CoV-2 in eventual incubation stages. The guest in isolation should undergo a nasopharyngeal swab at 7 and 14 days after entering the facility, before the final accommodation. The same indications were also given for guests who re-enter after hospitalization ${ }^{11}$.

The Puglia region, by a regional Provision with Protocol number AOO/ 005/ 251 of 25 $5^{\text {th }}$ March $2020{ }^{13}$, prohibited the access of new guests in semi-residential facilities and allowed the reintegration of patients temporarily absent for hospitalization, only with a negative swab issued by the hospital and, in any case, recommended that the returned patient be welcomed taken a dedicated area not in contact with the other guests of the semi-residential structure. In addition, the region denied the access of external visitors and family members during the emergency period. If the visit was necessary, a single visitor was allowed with a mask, who had carried out correct hand sanitization with soap and water for 60 seconds or with alcoholic sanitizers for 30 seconds. It is noteworthy that the region of Puglia emphasized the need to set up two dedicated areas in the structure: a special one in which to isolate suspected COVID-19 cases (guests with fever, cough, etc) and probable COVID-19 cases (doubtful oropharyngeal swab), and a second areas in which to isolate COVID-19 cases confirmed by laboratory, but that do not require hospitalization. For the latter, it was recommended the assistance of personnel equipped with PPE and informed about the measures to be taken to correctly wear and dispose of the aforementioned PPE after contact with an affected guest ${ }^{13}$.

On $9^{\text {th }}$ April 2020, with note protocol number 13,253, the Health Department of the Sicily region issues a "Vademecum for the prevention of the COVID-19 epidemic for fragile subjects assisted in the LTCF" ${ }^{14}$. This Vademecum contains behavioural rules to stem SARSCoV-2 contagion risk and provides periodic checks and screening on guests and staff through the swab or serological tests. The Vademecum also imposes daily control with traceability of body temperature and presence/absence of possible COVID-related symptoms between guests and staff of the structure. In addition, in each structure it will be necessary to identify a biocontainment officer who supervise all prevention procedure such as, for example, ensuring the traceability of health workers and all staff. In the same document is reported the obligation of the employees to be trained on the correct use of PPE, and the implementation of the facilities' cleaning. Among the countermeasures adopted, it is remarkable the absolute prohibition of visits from outside of relatives and acquaintances, the organization of recreational activities and the administration of meals in common areas ${ }^{14}$.

\section{ITALIAN HEALTH SYSTEM (ISS) REPORT IN LTCF}

From 24 ${ }^{\text {th }}$ March 2020, the ISS has started a survey about COVID-19 contagion in LTCF to monitor the situation, implementing control and prevention programs for healthcare-related infections. The data source consists of 3417 Italian LTCF. The study was based on a 29-question survey questionnaire aimed to acquire information about the situation from $1^{\text {st }}$ February to $5^{\text {th }}$ May 2020, and the procedures to reduce the risk of COVID-19 contagion. According to National Guarantor for Private Liberties (GNPL) Register, in Italy there are 4629 LTCF (public, affiliated, and private). The survey, to date, has involved 3292 LTCF (96\% on the total). From $25^{\text {th }}$ March to $27^{\text {th }}$ April, 1356 out of 3300 (41.3\%) 
of contacted structures answered the questionnaire. Most of the completed questionnaires came from Lombardia, Toscana, Veneto, Piemonte, Emilia Romagna. The response rate was $41 \%$, (from 0\% of Valle D'Aosta and Basilicata to over $50 \%$ of Molise, Sicilia, and Puglia). This variability was due to the limited time since sending the questionnaire and to the absolute number of structures present in the regions (Tab. I) ${ }^{6}$.

The health and care-workers operating in the LTCF were represented in mean by 2.5 doctors, 8.5 nurses, and 31.7 social health operators for each LTCF. 11\% of the structures declared no doctors among professional figures operating in the facility. Considering these three figures, each structure has an average of 42.4 operators (median of 33), with other professionals represented by physiotherapists, rehabilitation technicians, educators/animator, psychologists, for a total average of 5.4 operators for each facility.

Each structure reported 77 beds, in a range from 8 to 667 beds, with an average ratio of 2 beds per each operator (range 0.5-16.6). Considering only doctors and nurses, there are on average 8 beds per each operator (minimum of 0.6 and maximum of 42 ).

On $1^{\text {st }}$ February 2020, 97,521 residents lived in the 1356 LTCF surveyed, with an average of 72 residents per structure (range 7-632). Nine thousand one hundred and fifty-four residents died between $1^{\text {st }}$ of February to the date of filling in the questionnaire (26 March-5 May). The highest percentage of deaths has been registered in Lombardia (41.4\%), Piemonte (18.1\%) e Veneto (12.4\%). The mortality rate, calculated as the number of deceased out of total residents (residents sum on $1^{\text {st }}$ February and new entries from $1^{\text {st }}$ March), was equal to $9.1 \%$. Among died residents, 680 (7.4\%) were positive to the COVID-19 swab, while 3092 (33.8\%) had flu-like symptoms or pneumonia (regardless of swab execution). The mortality rate, considering the deaths of people positive to the test, was 0.7 per 100 residents. This value has increased up to $2.7 \%$ in the autonomous province of Trento. Mortality rate, considering deaths of residents with flu-like symptoms was $3.1 \%$, but increased up to $6.5 \%$ in Lombardia (Tab. II, Fig. 1).

Of the 5292 LTCF residents, from $1^{\text {st }}$ February to $5^{\text {th }}$ May 2020, 1342 have been hospitalized, with 4 hospitalizations per facility on average. The COVID-19 positive residents hospitalized from 1 February to date were $18.2 \%$ of the total, and residents with flu-like symptoms or pneumonia, regardless of swab execution, were $38.2 \%$ of the total (Fig. 2).

In each Italian region there were LTCF with subjects affected by SARS-CoV-2, with the highest attendance in Lombardia, Veneto, Toscana, Emilia Romagna e Piemonte.

Table I. Contacted facilities and answers obtained by Italian Regions (from Baldacci et al., 2012, mod.) ${ }^{3}$.

\begin{tabular}{|c|c|c|c|c|c|}
\hline \begin{tabular}{|l|} 
Regions \\
\end{tabular} & $\begin{array}{c}n . \\
\text { of LTCF }\end{array}$ & $\begin{array}{c}\% \\
\text { on total }\end{array}$ & $\begin{array}{l}\text { Called at } \\
\text { April } 27^{\text {th }}\end{array}$ & 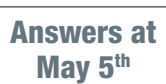 & $\begin{array}{c}\% \text { on total } \\
\text { contacts }\end{array}$ \\
\hline PIEMONTE & 608 & 17.8 & 608 & 249 & 41.0 \\
\hline VALLE D'AOSTA & 2 & 0.1 & 2 & 0 & 0.0 \\
\hline LOMBARDIA & 678 & 19.8 & 677 & 292 & 43.1 \\
\hline BOLZANO & 37 & 1.1 & 37 & 4 & 10.8 \\
\hline TRENTO & 54 & 1.6 & 51 & 15 & 29.4 \\
\hline VENETO & 520 & 15.2 & 520 & 148 & 28.4 \\
\hline FRIULI VENEZIA GIULIA & 70 & 2.0 & 70 & 39 & 55.7 \\
\hline LIGURIA & 120 & 3.5 & 116 & 20 & 17.2 \\
\hline EMILIA ROMAGNA & 348 & 10.2 & 278 & 128 & 46.0 \\
\hline TOSCANA & 319 & 9.3 & 319 & 200 & 62.7 \\
\hline UMBRIA & 50 & 1.5 & 42 & 16 & 38.1 \\
\hline MARCHE & 51 & 1.5 & 40 & 36 & 90.8 \\
\hline LAZIO & 207 & 6.1 & 192 & 79 & 41.1 \\
\hline ABRUZZO & 16 & 0.5 & 16 & 8 & 49.0 \\
\hline MOLISE & 6 & 0.2 & 6 & 4 & 66.7 \\
\hline CAMPANIA & 121 & 3.5 & 121 & 16 & 13.2 \\
\hline PUGLIA & 61 & 1.8 & 61 & 35 & 57.4 \\
\hline BASILICATA & 1 & 0.0 & 1 & 0 & 0.0 \\
\hline CALABRIA & 80 & 2.3 & 80 & 36 & 45.0 \\
\hline SICILIA & 52 & 1.5 & 39 & 24 & 61.5 \\
\hline SARDEGNA & 16 & 0.5 & 16 & 7 & 43.8 \\
\hline Total & 3417 & 100.0 & 3292 & 1356 & 41.2 \\
\hline
\end{tabular}


Table II. Total deceased with COVID-19 positive swabs and with flu-like symptoms, per region (from Baldacci et al., 2012, mod.) ${ }^{3}$.

\begin{tabular}{|c|c|c|c|c|c|}
\hline Italian Regions & $\begin{array}{c}\text { Deaths, } \\
\mathbf{n}\end{array}$ & $\begin{array}{c}\text { COVID-19 } \\
(+) \\
n(\%)\end{array}$ & $\begin{array}{c}\text { COVID-19 } \\
\text { symptomatic, } \\
n(\%)\end{array}$ & $\begin{array}{c}\text { Mortality } \\
\text { COVID-19 (+), } \\
\%\end{array}$ & $\begin{array}{c}\text { Mortality rate } \\
\text { COVID-19 } \\
\text { symptomatic, \% }\end{array}$ \\
\hline Piemonte & 1658 & $161(9.7)$ & $410(24.7)$ & 0.9 & 2.4 \\
\hline Lombardia & 3793 & $281(7.4)$ & $1807(47.6)$ & 1.0 & 6.5 \\
\hline PA Bolzano & 28 & $3(10.7)$ & $10(35.7)$ & 0.7 & 2.4 \\
\hline PA Trento & 99 & $33(33.3)$ & $45(45.5)$ & 2.7 & 3.7 \\
\hline Veneto & 1136 & $38(3.3)$ & $180(15.8)$ & 0.2 & 1.0 \\
\hline Friuli Venezia Giulia & 222 & $6(2.7)$ & $41(18.5)$ & 0.2 & 1.1 \\
\hline Liguria & 136 & $20(14.7)$ & $34(25)$ & 1.3 & 2.2 \\
\hline Emilia Romagna & 639 & $81(12.7)$ & $265(41.5)$ & 1.0 & 3.2 \\
\hline Toscana & 640 & $36(5.6)$ & $154(24.1)$ & 0.4 & 1.6 \\
\hline Umbria & 38 & $0(0)$ & $11(28.9)$ & 0.0 & 1.5 \\
\hline Marche & 160 & $13(8.1)$ & $59(36.9)$ & 0.9 & 4.3 \\
\hline Lazio & 158 & $1(0.6)$ & $28(17.7)$ & 0.0 & 0.6 \\
\hline Abruzzo & 47 & $1(2.1)$ & $0(0)$ & 0.2 & 0.0 \\
\hline Molise & 24 & $0(0)$ & $2(8.3)$ & 0.0 & 0.9 \\
\hline Campania & 50 & $6(12)$ & $13(26)$ & 0.9 & 2.0 \\
\hline Puglia & 111 & $0(0)$ & $4(3.6)$ & 0.0 & 0.2 \\
\hline Calabria & 75 & $0(0)$ & $1(1.3)$ & 0.0 & 0.1 \\
\hline Sicilia & 73 & $0(0)$ & $11(15.1)$ & 0.0 & 1.0 \\
\hline Sardegna & 67 & $0(0)$ & $17(25.4)$ & 0.0 & 2.8 \\
\hline Total & 9154 & $680(7.4)$ & 3092 (33.8) & 0.7 & 3.1 \\
\hline
\end{tabular}

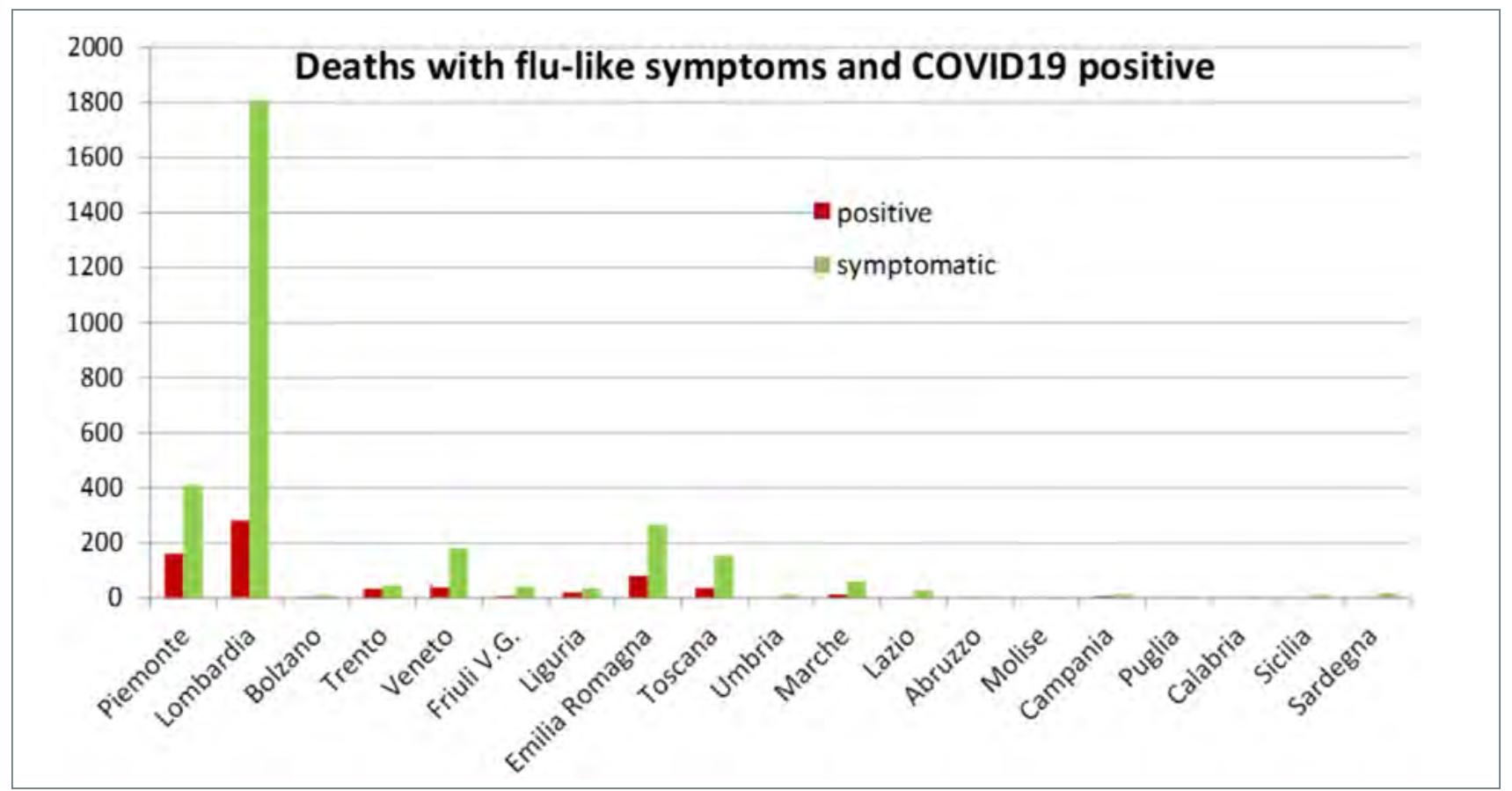

Figure 1. Resident deceases in COVID-19 positive swabs and flu-like symptoms, per region (from ISS, 2020) ${ }^{6}$.

Concerning the question on the main difficulties during the epidemic, 1259 facilities answered, 972 (77.2\%) reported an absence of PPE, 263 (20.9\%) stated a lack of information received for infection containment. 123
(9.8\%) facilities described medication lack, 425 (33.8\%) absence of healthcare staff, and 157 (12.5\%) difficulty in transferring positive patients to hospital facilities. Finally, 330 declared (26.2\%) difficulty in isolating COVID-19 


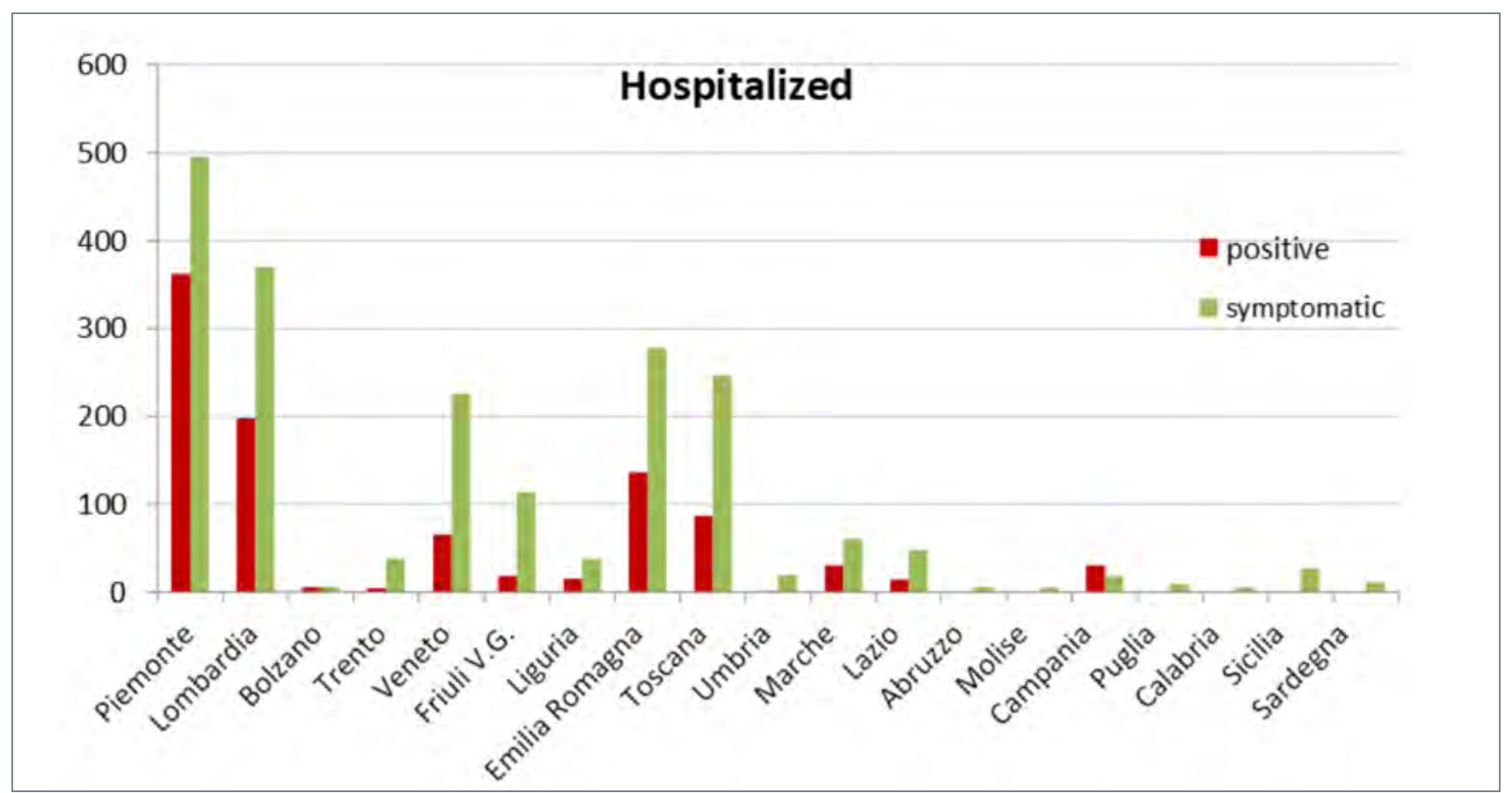

Figure. 2. Hospitalized residents with COVID-19 positive swabs and flu-like symptoms, per region (from ISS, 2020) ${ }^{6}$.

positive subjects, and 282 reported the impossibility of swab execution. These data concern $52.1 \%$ of facilities that have answered in this way because this option was added on $8^{\text {th }}$ April. 272 facilities (21.6\%) declared other types of difficulties. Main difficulties concern lack of PPE and patients' management.

According to DPCM 08/03/2020, except one, 1346 facilities that answered declared visit's interdiction of relatives/caregivers to residents. Visits were allowed only in case of serious deterioration of residents' health conditions or if in the terminal life phase. Except 6, all LTCF declared having adopted alternative communication forms with family/caregivers.

Regarding the SARS-COV-2 positivity among staff, 1320 LTCF answered: 278 (21.1\%) declared SARS-COV-2 positivity among their staff. Regions with highest percentages were: Bolzano (50.0\%) and Trento (46.7\%) followed by Lombardia (40.0\%), Piemonte (25.0\%), Marche (23.5\%), Emilia Romagna (18.1\%), Veneto (16.6\%), Liguria (15.8\%), Friuli Venezia Giulia (12.8\%), Toscana (12.4\%). 1239 facilities (92.9\%), out of 1334 that answered this question, declared to have written procedure for outbreak management, 95 (7.1\%) declared they did not.

Six hundred and fifty LTCF (48.1\%) out of 1351 declared to have a single room for subjects with confirmed or suspected infection; 30.7\% ( $n=145)$ can isolate patients' groups, few have a structure dedicated to isolation, $8.1 \%$ adopted other isolation forms and 3.1\% adopted a combination of these isolation forms; 104 structures $(8 \%)$ reported no possibility to isolate infected patients.
The facilities that carried out a training program for health personnel on the correct use of PPE were the 93.3\%. Only $6.7 \%$ LCTF did not performed any training on this topic.

The question on flu vaccination coverage among residents was added on $30^{\text {th }}$ March, and 1045 LTCF answered. In these latest, an average of vaccination coverage of $86 \%$ of the residents was found, with $21.3 \%$ of LTCF reporting a 100\% vaccination coverage, while 5 facilities reported $0 \%$ vaccination coverage. No peculiar reasons neither specific characteristic of LCTF could explain the causes of no vaccination.

\section{THE ISS LEGISLATION}

The ISS developed legislation (on $17^{\text {th }}$ April 2020) on prevention measures, with the main arrangements are listed below ${ }^{15}$ :

- Organization measures to prevent the entry of COVID-19 cases

- Identify a COVID-19 healthcare referent that provides interventions and guarantees relationships with relevant institutions (Department of Prevention, Health Districts, and ASL);

- Manage communication with staff, residents and their family;

- Reinforce standard precautions.

- Setting the facility to manage suspected/probable/ confirmed COVID-19 cases

- $\quad$ During the emergency, deny access of family patients and caregivers;

- $\quad$ Prevent access to symptomatic persons; 
- Limit new resident entrances to LTCF after health assessment and swab;

- Reduce residents' hospitalization with specialist visits and instrumental examinations;

- Isolate area for new admissions;

- $\quad$ Suspend group activities in the common spaces within the facility;

- Permit access of health workers, if possible, but avoiding musters;

- $\quad$ Claim the use of surgical masks and correct hand hygiene for all staff;

- Distribute Personal Protective Equipment (PPE), hydro-alcoholic solutions, soap, etc. and procurement of contactless thermometers.

- Suspected case management (isolation pending swab results) or confirmed case management

- The suspected COVID-19 case, immediately placed in isolation, must be reported to the public hygiene service and undergo to a nose-pharyngeal swab for SARS-CoV-2. If positive, the Department of Prevention will have to verify the possibility of isolation into the same structure. If not possible, the patient will be transferred to a hospital or a more suitable structure, for isolation, further clinical evaluation, and necessary treatment, such as in a facility dedicated to COVID-19 patients. Also, careful sanitization of the environment where the resident lived and where he was examined must be carried out immediately. In case of risk identification, the exposed subjects must be considered contacts of a COVID19 case and followed the reporting, surveillance, and quarantine procedures established by the ASL according to the Ministerial circular of 25 March $2020{ }^{16}$;

- In larger facilities, provide dedicated areas and paths that can ensure separation between "clean" and "dirty" areas and carry out specific procedures for temporary isolation;

- In cases of suspected/probable/confirmed infection, USCAs (Special Continuity Units) must be activated with the advice/collaboration of infectious disease specialists;

- Reduce contacts of residents with suspected/ probable/confirmed cases of COVID-19.

- Measures for managing roommates and other close contacts of a COVID-19 case $^{16}$.

- Staff training for the proper adoption of standard precautions and isolation measures

- Characteristics of SARS-CoV-2 infection and COVID-19 disease;

- $\quad$ Standard precautions for assistance to all patients: hand and respiratory hygiene;

- Precautions in preventing COVID-19 transmission by contact and droplets while assisting suspected or probable/confirmed cases of COVID-19;

- $\quad$ Precautions for the prevention of airborne diseases;

- $\quad$ Proper use of medical devices and PPE;

- Behaviors to be implemented during breaks and meetings to reduce virus transmission ${ }^{16}$.

\section{EUROPEAN LTCF GUIDELINES}

The European Centre for Disease, Prevention, and Control (ECDC) drew up measures for COVID-19 management and infection prevention in LTCF. Some of the most important recommendations are ${ }^{17}$ :

- visits to COVID-19 residents have to be limited. Visitors should wear PPE. If they don't have PPE but a surgical mask, they have to observe a distance of at least one meter from residents. Moreover, it's suggested to draw up a register for contacts tracing. People who visit a confirmed COVID-19 case should self-monitor any symptoms of COVID-19 for 14 days after the visit;

- appointing a team or at least one full-time staff member in each structure to be the lead for COVID-19;

- updating business continuity plans, if staff members become ill or in self-isolation;

- monitoring local and national public health sources to understand COVID-19 activity in the community;

- placing signs at every entrance representing symptoms compatible with COVID-19 ${ }^{13}$, informing visitors with any of these symptoms not to enter the LTCF;

- guaranteeing that all people at the LTCF and enter the facility perform a correct hand hygiene procedure;

- assessing new and returning residents for symptoms compatible with COVID-19;

- ensuring that staff members with symptoms compatible with COVID-19 do not go to work, and contact a pre-designated telephone number or a contact point to inform for symptoms;

- restricting non-essential visits and increasing physical distancing measures. Ensuring that staff members with symptoms of respiratory infection stop working and self-isolate;

- if possible, LTCF should identify dedicated staff to care for residents with suspected/confirmed COVID-19 to reduce the likelihood of transmission to other residents;

- training all staff to the correct use of PPE;

- ECDC guidance recommends laboratory testing samples from all suspected cases after the individuation of a suspected case and testing all staff and residents if there is a laboratory-confirmed case; 
- when a LTCF patient shows COVID-19 signs or symptoms, public health authorities/healthcare services should be contacted for notification, assessment, and measures on testing and possible transfer to an acute care hospital performed;

- residents with COVID-19 symptoms not requiring hospitalization should be isolated in single rooms with a separate bathroom. If more cases, they must be placed in a separate ward or section of the facility with dedicated staff. With a small number of cases, patients should preferably be admitted to an isolation room with a dedicated toilet, if available. If feasible, patients must be placed in single rooms with negative pressure and anteroom;

- guaranteeing that all LTCF staff are conscious of residents showing symptoms or having tested positive for COVID-19;

- healthcare staff and other workers coming into contact with residents who have respiratory infections should wear a surgical mask or a Filtering FacePiece type 2 (FFP2) respirator if available, eye protection (i.e. visor or safety goggles), a gown and gloves;

- regular cleaning, followed by disinfection, is recommended. Cleaning personnel should use hospital active disinfectants against viruses for all common care facility areas and residents' rooms (furniture and touched surfaces). If there is a shortage of hospital disinfectants, surfaces may be decontaminated with $0.05 \%$ sodium hypochlorite (dilution 1:100 if household bleach at an initial concentration of $5 \%$ is used) after cleaning with a neutral detergent. However, it should be noted that no data are available on the effectiveness of this approach against SARS-CoV-2. Surfaces that do not tolerate sodium hypochlorite may be cleaned with a neutral detergent, followed by a $70 \%$ concentration of ethanol ${ }^{18,19}$.

\section{ITALIAN MEASURES FOR THE FUTURE}

The Decree of $17^{\text {th }}$ May 2020 stated that visitors or relatives' access to LTCF, rehabilitation centers, care facilities for older persons must be limited to those cases which are covered by the Health Department, bound to adopt the necessary measures to prevent any infection ${ }^{12}$.

The Lombardia region arranged the re-opening of LTCF with the DGR XI/3226, which established the carrying out of serological tests and swabs for the new accesses to such facilities, people tested positive taken into specialized COVID centers, screenings and training courses for staff. Hospital visits are limited only to necessity reasons and must be authorized by the administration. The new procedures require meticulous monitoring aimed at ruling out the presence of suspected symptoms of COVID19 or exposure to COVID cases in the previous 14 days and the possibility of home isolation. Nasopharyngeal swabs and serologic tests are provided for people at home, continuing the home isolation along the period between the tests and the arrival date to the center. Access to the center is only permitted after 14 days of home isolation with negative tests before and after the confinement. COVID fully recovered patients who are going to become LTCF guests are allowed; clinically healed patients awaiting last negative tests, are admitted into specific care centers. Serological tests and swabs are also provided for guests who periodically need outpatient services. Specific training courses, screenings, and monitoring of the health of the crew and service providers are set up, as well as a denied access to the healthcare facility in case of symptoms ${ }^{20}$.

The Veneto region, from $1^{\text {st }}$ June 2020, newly allowed visitors to enter the LTCF by adopting preventive measures similarly to Lombardia. Visitors undergo quick tests. Ultimately, taking into account the progression of the disease, Veneto established a vaccination plan to all the people attending LTCF, since flu symptoms occurring early in the illness are quite similar to COVID-19 symptoms. Flu vaccine could make COVID diagnoses easier, isolating the new suspected cases more rapidly. Pneumococcal conjugate vaccine is provided for those that have not been vaccinated ${ }^{21}$.

\section{CONCLUSIONS}

SARS-COV-2 pandemic had a grievous impact on LTCF in Italy, causing a severe and underestimated death percentage among the frailest subjects of the population. LTCF has become "contagion prisons", no more safe places. The new national and European guidelines about the management of contagion are centered on the highest safety for the reception and provided services, so that LTCF are again placed in which the weakest can put their trust to be assisted in their daily needs. Containment measures should be particularly optimized, especially in anticipation of another possible pandemic recurrence. The persons 65 years and older may represent a specific cluster of high-risk patients for developing COVID-19 with rapidly progressive clinical deterioration ${ }^{22}$. Lipsitz et al. ${ }^{23}$ described an interventional program including mandatory testing of all residents, and staff, associated with positive outcomes. However, the same authors concluded that, without a randomized controlled study, it is difficult to settle that the benefits were attributable solely to the intervention ${ }^{23}$. This review summarizes the Italian management 
of COVID-19 older patients in LTCF. Surely a comparison among different systems could help to achieve more information in the standardization of procedures. In fact, identifying flaws would be of interest and necessary to perfect and optimize epidemic management as much as possible, especially in the LTCF.

\section{Ethical consideration}

Not applicable.

\section{Acknowledgement}

This research did not receive any funding from agencies in the public, commercial, or not-for-profit sectors.

\section{Conflict of interest}

The Authors declare no conflict of interest.

\section{Funding}

This research did not receive any specific grant from funding agencies in the public, commercial, or not-forprofit sectors.

\section{References}

1 ISS. Characteristics of COVID-19 patients dying in Italy Report based on available data on July $22^{\text {th }}, 2020$ (https:// www.epicentro.iss.it/en/coronavirus/sars-cov-2-analysisof-deaths, Accessed 4 January 2021).

2 Maio S, Baldacci S, Simoni M, et al.; ARGA Study Group. Impact of asthma and comorbid allergic rhinitis on quality of life and control in patients of Italian general practitioners. J Asthma 2012;49:854-861. https://doi.org/10.3109/027 70903.2012.716471

3 Baldacci S, Maio S, Simoni M, et al.; ARGA study group. The ARGA study with general practitioners: impact of medical education on asthma/rhinitis management. Respir Med 2012;106:777-785. https://doi.org/10.1016/j. rmed.2012.02.013

4 D.P.C.M. 22/12/1989. on "Act of direction and coordination of the administrative activity of the regions and autonomous provinces concerning the construction of residential health facilities for non-self-sufficient elderly who cannot be assisted at home or in semi-residential services [Atto di indirizzo e coordinamento dell'attività amministrativa delle regioni e province autonome concernente la realizzazione di strutture sanitarie residenziali per anziani non autosufficienti non assistibili a domicilio o nei servizi semiresidenziali]" (https://www.gazzettaufficiale.it/ atto/serie_generale/caricaDettaglioAtto/originario?atto. dataPubblicazioneGazzetta=1990-01-03\&atto.codiceRedazionale=089A5965\&elenco30giorni=false, Accessed 1 February 2021).

5 D.P.R. 27/04/1978 on Implementation regulation of art. 27 of Law no. 118, in favor of the disabled and civil disabled, in the matter of architectural barriers and public transport." [Regolamento di attuazione dell'art. 27 della L. 30 marzo 1971 , n. 118, a favore dei mutilati e invalidi civili, in materia di barriere architettoniche e trasporti pubblici.] (https:// www.normattiva.it/uri-res/N2Ls?urn:nir:presidente.repubblica:decreto:1978-04-27;384, Accessed 8 February 2021).

6 ISS. A survey on COVID-19 infection in long-stay residential care homes. COVID-19 Epidemic, National updating May 5, 2020 (https://www.epicentro.iss.it/en/coronavirus/ sars-cov-2-survey-rsa. Accessed 8 January 2021).

7 Lombardia Region Decree no. XI/ 2906, March 8, 2020 (https://www.regione.lombardia.it/wps/wcm/ connect/5e0deec4-caca-409c-825b-25f781d8756c/ $\mathrm{DGR}+2906+8+$ marzo+2020. pdf?MOD=AJPERES\& CACHEID=ROOTWORKSPACE-5e0deec4-caca-409c-82 5b-25f781d8756c-n7b4|OB, Accessed 10 January 2021).

8 D.P.G.R. no. 34 Piemonte Region Dated 21.03.2020 (https://www.regione.piemonte.it/web/sites/default/files/ media/documenti/2020-03/decreto_presidente_della_giunta_regionale_n._34_-_21_marzo_2020.pdf, Accessed 4 February 2021).

9 D.G.R. no. 28 Toscana Region Dated 07.03.2020 (http:// www301.regione.toscana.it/bancadati/atti/Contenuto. $\mathrm{xml}$ ?id=5246964\&nomeFile=Ordinanza_del_Presidente_n.13_del_16-03-2020, Accessed 27 December 2020).

10 Ordinance no. 16 Campania Region Dated 13.03.2020 (Regione: Ordinanza n. 16 del 13 marzo 2020 | IFEL Campania).

11 Regional Directorate General for the Protection of Health, note prot. no. 0260863 Campania region Dated 4.06.2020 (Coronavirus - Note DG Tutela della Salute - Informazioni Regione Campania).

12 D.P.C.M. Dated 17/05/2020 (http://www.governo.it/sites/ new.governo.it/files/DPCM_20200517.pdf, Accessed 15 January 2021).

13 Regional Provision Prot. no. AOO/ 005/ 251 Puglia Region Dated 25.03.2020 (https://www.sanita.puglia. it/documents/36044/102993901/disposizione+rsa/ aa37af6c-550a-496f-8f68-fcd40dc54f3e).

14 Health Department Vademecum prot. no.13253, Sicily Region Dated 9.04.2020 (Nota prot. n. 15977 del 29 Aprile 2020 Recepimento Circo.pdf (regione.sicilia.it).

15 ISS Working Group on Infection, Prevention and Control COVID-19. Interim guidance for prevention and control of SARS-CoV-2 infection in long term care facilities. Version April 17, 2020 (https://www.iss.it/documents/20126/0/Rapporto+ISS+COVID-19+n.4-2020_Rev.+EN.pdf/ade7a16dab1e-dfa8-51ce-862aedb1ed66?t=1589367338007, Accessed 20 December 2020).

16 D.L 25/03/2020 n. 19 on " Urgent measures to deal with the epidemiological emergency from COVID-19" [Misure urgenti per fronteggiare l'emergenza epidemiologica da COVID-19] (https://www.interno.gov.it/it/amministrazione-trasparente/disposizioni-generali/atti-generali/ atti-amministrativi-generali/circolari/circolare-26-marzo-2020-decreto-legge-25-marzo-2020-n-19-recante-misure-urgenti-fronteggiare-lemergenza-epidemiologica-covid-19, Accessed 29 January 2021). 
17 European Centre for Disease Prevention and Control. Infection prevention and control for COVID-19 in healthcare settings - third update, 13 May 2020. ECDC: Stockholm; 2020 (https://www.ecdc.europa.eu/sites/default/files/documents/Infection-prevention-control-for-the-care-of-patients-with-2019-nCoV-healthcare-settings_third-update. pdf, Accessed 3 February 2021).

18 US Centers for Disease Control and Prevention (UDC). Strategies to prevent the spread of COVID-19 in long term care facilities (LCTF) 2020.

19 European Centre for Disease Prevention and Control. Surveillance of COVID-19 in long-term care facilities in the EU/ EEA, 19 May 2020. Stockholm: ECDC; 2020. ๑European Centre for Disease Prevention and Control, Stockholm, 2020 (https://www.ecdc.europa.eu/sites/default/files/documents/covid-19-long-term-care-facilities-surveillanceguidance.pdf, Accessed 20 January 2021).
20 D.G.R. Lombardia Region, no. Xl/3226 Dated 09.06.2020 (https://anci.lombardia.it/documenti/10076-DGR\%20 3226\%20DEL\%209_6_2020.pdf, Accessed 4 January 2021).

21 D.G.R. no. 898 Veneto Region on Guidelines for LCTF and similar Dated 22.06.2020 (https://www.regione.veneto.it/ article-detail?articleld=4897021, Accessed 3 December 2020).

22 Perrotta F, Corbi G, Mazzeo G, et al. COVID-19 and the elderly: insights into pathogenesis and clinical decision-making. Aging Clin Exp Res 2020;32:1599-1608. https://doi. org/10.1007/s40520-020-01631-y

23 Lipsitz LA, Lujan AM, Dufour A, et al. Stemming the tide of COVID-19 infections in Massachusetts nursing homes. J Am Geriatr Soc 2020;68:2447-2453. https://doi. org/10.1111/jgs.16832 